\title{
Finite Groups Whose Character Graphs Associated with Codegrees
}

\author{
Have No Triangles \\ Huan Xiong \\ Université de Strasbourg, CNRS, IRMA UMR 7501, F-67000 Strasbourg, France \\ E-mail: xiong@math.unistra.fr
}

\begin{abstract}
Motivated by Problem 164 proposed by Y. Berkovich and E. Zhmud' in their book "Characters of Finite Groups", we give a characterization of finite groups whose irreducible character codegrees are prime powers. This is based on a new kind of character graphs of finite groups associated with codegrees. Such graphs have close and obvious connections with character coedgree graphs. For example, they have the same number of connected components. By analogy with the work of finite groups whose character graphs (associated with degrees) have no triangles, we conduct a result of classifying finite groups whose character graphs associated with codegrees have no triangles in the latter part of this paper.
\end{abstract}

2010 Mathematics Subject Classification: 20C15

Keywords: finite group, character graph, codegree

\section{Introduction}

Let $G$ be a nontrivial finite group throughout the paper. Let $\chi$ be a character of $G$ and $a(\chi):=\frac{|G: k e r \chi|}{\chi(1)}$ the so-called character codegree of the character $\chi$. Y. Berkovich and E. Zhmud' proposed a problem in [1] which is to study a finite group $G$ such that $a(\chi)$ is a prime power for every $\chi \in \operatorname{Irr}(G)$ (see Problem 164 of [1, Page 306). In this paper we solve this problem by giving a characterization of such groups.

Theorem 1.1 Let $G$ be a finite group. Then all the codegrees of irreducible characters of $G$ are prime powers if and only if $G$ is a p-group or a Frobenius group whose order has exactly two prime divisors.

Our method is based on a new kind of character graph $\Gamma(G)$ of a finite group $G$. 
Definition 1.2 The graph $\Gamma(G)$, which is called character graph of $G$ associated with codegrees, is defined as follows: the vertex set is $\operatorname{Irr}(G) \backslash\left\{1_{G}\right\}$ and there is an edge between $\chi, \psi \in \operatorname{Irr}(G) \backslash$ $\left\{1_{G}\right\}$ if and only if the greatest common divisor $\operatorname{gcd}(a(\chi), a(\psi)) \neq 1$.

There are several kinds of graphs associated with irreducible characters and degrees of finite groups. They were received far-reaching attention in the last more than twenty years. For detailed information on these graphs, we refer to [12, 13], and a survey article [5].

Based on the idea of character degree graphs of finite groups, G. Qian, Y.Wang, and H. Wei define the character codegree graph $\Delta(G)$ of a finite group $G$ in [8]. Specifically, the graph $\Delta(G)$ is defined as follows: the vertices of $\Delta(G)$ are the primes dividing the codegree of some nonprincipal irreducible character of $G$, and the vertices $p$ and $q$ are connected by an edge if and only if there exists a codegree of some nonprincipal irreducible character of $G$ divisible by $p q$. In [8], the authors develop some properties of the graph $\Delta(G)$. For example, they show that if $\Delta(G)$ is connected, then its diameter is at most 3. Also, they show that $\Delta(G)$ has at most 2 connected components, and that $\Delta(G)$ is not connected if and only if $G$ is Frobenius or 2-Frobenius.

Just as there are close connections between the character graph and the character degree graph of a finite group, the character graph associated with codegrees and the character codegree graph of a finite group are closely related. Obviously, the two graphs have the same number of connected components. Furthermore, by Corollary 3.2 in [5] and results about $\Delta(G)$ in [8], we have the following result:

Theorem 1.3 Let $G$ be a finite group and let $\Gamma(G)$ be the character graph of $G$ associated with codegrees. Then the following statements hold:

(1) $\Gamma(G)$ has at most 2 connected components;

(2) If $\Gamma(G)$ is connected, then its diameter is at most 4; and

(3) $\Gamma(G)$ is not connected if and only if $G$ is Frobenius or 2-Frobenius.

Among all kinds of shapes of character graphs (or character degree graphs) of finite groups, having no triangles is probably a distinguishing one. Finite groups whose character graphs associated with degrees have no triangles are characterized (see [11] for the solvable case and [6] for the nonsolvable case). We conduct an analogous work of investigating finite groups whose character graphs associated with codegrees have no triangles in the latter part of this paper. Indeed, we get a classification of such groups:

Theorem 1.4 Let $G$ be a nontrivial finite group whose character graph associated with codegrees has no triangles. Then $G$ is isomorphic to one of the following groups: 
- The cyclic group of order 2 or 3 ;

- The alternating group $A_{4}$;

-The symmetric group $S_{3}$;

- The dihedral group $D_{10}$ of order 10; and

- The nonabelian group $F_{7,3}$ of order 21 .

Conversely, all these groups' character graphs associated with codegrees have no triangles.

With the list of finite groups whose character graphs associated with codegrees have no triangles, we get the following obvious result:

Corollary 1.5 Let $G$ be a finite group whose character graph associated with codegrees has no triangles. Then $G$ is solvable.

\section{Preliminaries}

In this section, we present some preliminary results needed later.

By Lemma 2.1 in [8], we have the following result:

Lemma 2.1 Let $\chi \in \operatorname{Irr}(G)$.

(1) For any $N \unlhd G$ with $N \leq k e r \chi$, $\chi$ may be viewed as an irreducible character of $G / N$. The codegree $a(\chi)$ of $\chi$ is the same whenever $\chi$ is seen as an irreducible character of $G$ or $G / N$. Furthermore, $a(\chi)$ is independent of the choice of such $N$. In particular, $\Gamma(G / N)$ is a subgraph of $\Gamma(G)$.

(2) If $M$ is a subnormal subgroup of $G$ and $\psi$ is an irreducible constituent of $\chi_{M}$, then $a(\psi)$ divides $a(\chi)$.

Lemma 2.2 Let $G=H \times K, \chi \in \operatorname{Irr}(H)$, and $\psi \in \operatorname{Irr}(K)$. If $\operatorname{gcd}(|H|,|K|)=1$, then $a(\chi \times \psi)=a(\chi) a(\psi)$.

Proof. We first claim $\operatorname{ker}(\chi \times \psi)=\operatorname{ker} \chi \times \operatorname{ker} \psi$. Indeed, $\operatorname{ker}(\chi \times \psi)=H_{1} \times K_{1}$ for some $H_{1} \leq H$ and $K_{1} \leq K$ by Corollary 8.20 of [9]

It is easy to see that $H_{1} \leq k e r \chi$ and $K_{1} \leq k e r \psi$. Thus $\operatorname{ker}(\chi \times \psi) \leq k e r \chi \times k e r \psi$. Obviously, $\operatorname{ker}(\chi \times \psi) \geq \operatorname{ker} \chi \times \operatorname{ker} \psi$. Thus the claim holds.

Now $a(\chi \times \psi)=\frac{|G: k e r(\chi \times \psi)|}{\chi(1) \psi(1)}=\frac{|H: k e r \chi|}{\chi(1)} \cdot \frac{\mid K: \text { ker } \psi \mid}{\psi(1)}=a(\chi) a(\psi)$.

Corollary 2.3 Let $G$ be a nilpotent group. Suppose $G=P_{1} \times P_{2} \times \cdots \times P_{s}$ where $P_{i} \in$ Syl $_{p_{i}}(G)$ for $i=1,2, \cdots s$. If $\theta_{i} \in \operatorname{Irr}\left(P_{i}\right)$ where $i=1,2, \cdots s$, then $a\left(\theta_{1} \times \theta_{2} \times \cdots \times \theta_{s}\right)=$ $a\left(\theta_{1}\right) a\left(\theta_{2}\right) \cdots a\left(\theta_{s}\right)$. 
Theorem A of [8] implies the following result:

Lemma 2.4 For any prime $p|| G \mid$, there exists $\chi \in \operatorname{Irr}(G)$ such that $p \mid a(\chi)$.

We also need Theorem 8.17 of [3]:

Lemma 2.5 Let $\chi \in \operatorname{Irr}(G)$ and suppose $p \nmid(|G| / \chi(1))$ for some prime $p$. Then $\chi(g)=0$ whenever $p \mid o(g)$.

The following result is well-known (see [10]):

Lemma 2.6 If $G$ is a Frobenius group with Frobenius kernel $N$ and Frobenius complement $H$, then $N$ is a nilpotent group. Furthermore, if $H$ has even order then $N$ is abelian.

\section{Finite Groups Whose All Character Codegrees Are Prime Powers}

In this section, we prove Theorem[1.1 and thus solve Problem 164 proposed by Y. Berkovich and E. Zhmud' in 1 by characterizing finite groups all of whose irreducible character codegrees are prime powers.

Proof of Theorem 1.1 By Theorem 1.3, we can easily check that if $G$ is a $p$-group or a Frobenius group whose order has exactly two prime divisors, then all the irreducible character codegrees of $G$ are prime powers.

Conversely, suppose that $G$ is a finite group whose all character codegrees are prime powers. If $\Gamma(G)$ is connected, then all the irreducible character codegrees of $G$ must be powers of a fixed prime number. Thus, by Lemma 2.4, $|G|$ has only one prime divisor. This means that $G$ is a $p$-group.

If $\Gamma(G)$ is not connected, then $\Gamma(G)$ have two connected components by Theorem 1.3 , Since all character codegrees of $G$ are prime powers, it follows that $|G|$ has exactly two prime divisors by Lemma 2.4. Thus $G$ can not be a 2-Frobenius group. By Theorem 1.3 , $G$ is a Frobenius group and $|G|$ has exactly two prime divisors.

Theorem 1.1 has a direct corollary:

Corollary 3.1 If $G$ is a finite group whose all character codegrees are prime powers, then $G$ is solvable. 


\section{Finite Groups Whose Character Graphs Associated with Codegrees Have No Triangles}

We first investigate the character graphs associated with codegrees of finite simple groups.

Lemma 4.1 Let $G$ be a finite simple group. Then $\Gamma(G)$ is a complete graph.

In particular, if $\Gamma(G)$ has no triangles, then $G$ is a cyclic group of order 2 or 3 .

Proof. Let $1_{G} \neq \theta, \psi \in \operatorname{Irr}(G)$. Since $G$ is a finite simple group, it follows that $k e r \theta=1$, which means that $a(\theta)=|G: \operatorname{ker} \theta| / \theta(1)=|G| / \theta(1)$. Similarly, we have $a(\psi)=|G: \operatorname{ker} \psi| / \psi(1)=$ $|G| / \psi(1)$. Also, $\theta(1)^{2}<|G|, \psi(1)^{2}<|G|$ since $\sum_{\chi \in \operatorname{Irr}(G)} \chi(1)^{2}=|G|$. Therefore $\theta(1) \psi(1)<|G|$, which means there exists some prime $p$ and some positive integer $k$ such that $p^{k}|| G \mid$ and $p^{k} \nmid \theta(1) \psi(1)$. Then we see that $p \mid a(\theta)$ and $p \mid a(\psi)$, which means that $a(\chi)$ and $a(\psi)$ are not coprime and there is an edge between any two vertices in $\Gamma(G)$, i.e., $\Gamma(G)$ is a complete graph.

If $G$ is a finite simple group such that $\Gamma(G)$ has no triangles. By the above argument, $\Gamma(G)$ is a complete graph. Therefore, $G$ contains at most 2 nonprincipal irreducible characters, which implies that $G$ contains at most 3 conjugacy classes. Thus $G$ is a cyclic group of order 2 or 3 .

Now we study finite abelian groups whose character graphs associated with codegrees have no triangles.

Lemma 4.2 Let $G=C_{n}$ be a finite cyclic group of order $n$. Then for any positive integer $d \mid n$, $|\{\chi \in \operatorname{Irr}(G) \mid a(\chi)=d\}|=\varphi(d)$, where $\varphi$ is the well-known Euler's totient function such that $\varphi(d)=\mid\{m \mid 0<m \leq d$ and $\operatorname{gcd}(m, d)=1\} \mid$.

Proof. Let $G=\langle g\rangle$ and let $\varepsilon_{n}$ be a primitive $n$th root of unity. For any $1 \leq i \leq n$, set $\chi_{i}\left(g^{k}\right)=\varepsilon_{n}^{k i}$. Then $\chi_{i}(1 \leq i \leq n)$ are precisely all the irreducible characters of $G$. Moreover, there are exactly $\varphi(n)$ irreducible characters $\chi_{i}$ of $\mathrm{G}$ such that $k e r \chi_{i}=1$. Notice that for any $1 \leq i \leq n, \chi_{i}(1)=1$. Thus $a\left(\chi_{i}\right)=\left|G: \operatorname{ker} \chi_{i}\right| / \chi_{i}(1)=\left|G: \operatorname{ker} \chi_{i}\right|$. For any positive integer $d \mid n, a\left(\chi_{i}\right)=d$ is equivalent to either $\mid$ ker $\chi_{i} \mid=\frac{n}{d}$ or ker $\chi_{i}=\left\langle g^{d}\right\rangle$. Let $N_{d}=\left\langle g^{d}\right\rangle$. Then ker $\chi_{i}=N_{d}$ if and only if $\chi_{i}$ can be seen as a faithful irreducible character of $G / N_{d}$. Since the number of faithful irreducible characters of $G / N_{d}$ is $\varphi(d)$, we have $|\{\chi \in \operatorname{Irr}(G) \mid a(\chi)=d\}|=$ $\varphi(d)$.

Lemma 4.3 Let $G$ be an abelian group such that $\Gamma(G)$ has no triangles. Then $G$ is a cyclic group of order 2 or 3 . 
Proof. By Corollary 2.3 and Lemma 4.2 we may assume that $G$ is a $p$-group. Furthermore, if $|G|>3$, then $G$ has three distinct nonprincipal irreducible characters whose codegrees have common divisor $p$ by Lemma 4.2 and thus $\Gamma(G)$ has a triangle, a contradiction. Thus $G$ is a cyclic group of order 2 or 3.

Next we give reduction results about finite groups whose character graphs associated with codegrees have no triangles.

Lemma 4.4 Let $G$ be a nontrivial finite group such that $\Gamma(G)$ has no triangles. If $N$ is a maximal normal subgroup of $G$, then $|G / N|=2$ or $3, G^{\prime}=N$, and $N$ is the unique maximal normal subgroup of $G$.

Proof. If $G^{\prime}=1$, then the lemma holds by Lemma 4.3. So we may assume that $G$ is nonabelian. Note that $\Gamma(G / N)$ is a subgraph of $\Gamma(G)$. Since $\Gamma(G)$ has no triangles, it follows that $|G / N|=2$ or 3 by Lemma 4.1. Thus $G^{\prime} \leq N$. But since $\Gamma\left(G / G^{\prime}\right)$ also has no triangles, we have $\left|G / G^{\prime}\right|=2$ or 3 by Lemma 4.3. Thus $G^{\prime}=N$.

If $M$ is another maximal normal subgroup of $G$, then we have $M=G^{\prime}=N$ by the above argument. This means that $N$ is the unique maximal normal subgroup of $G$.

Lemma 4.5 Let $G$ be a nonabelian finite group such that $\Gamma(G)$ has no triangles. If $G$ has a normal subgroup $N$ such that $|G: N|=3$, then $G$ is isomorphic to the alternating group $A_{4}$ or the nonabelian group $F_{7,3}$ of order 21 .

Proof. Note that $N \neq 1$ since $G$ is nonabelian. Let $\operatorname{Irr}(G / N)=\left\{\chi_{1}=1_{G}, \chi_{2}, \chi_{3}\right\}$ and $P \in \operatorname{Syl}_{3}(G)$. By Lemma 4.4, we may assume that $G^{\prime}=N$ and that $N$ is the unique maximal normal subgroup of $G$.

Step 1. We claim that $3 \nmid|N|$ and thus $|P|=3$. Otherwise, suppose that 3||$N \mid$. Then by Lemma 2.4, there exists $\theta \in \operatorname{Irr}(N)$ such that $3 \mid a(\theta)$. Let $\chi$ be an irreducible constituent of $\theta^{G}$. Then $3 \mid a(\chi)$ by Lemma 2.1. Since $3 \mid a(\theta)$, we have $\theta \neq 1_{N}$. Thus $\chi_{N} \neq 1_{N}$ and $\chi \notin \operatorname{Irr}(G / N)$. Therefore we obtain $\chi, \chi_{2}$, and $\chi_{3}$ which are three distinct irreducible characters of $G$ and $3|a(\chi), 3| a\left(\chi_{2}\right), 3 \mid a\left(\chi_{3}\right)$. Then it follows that $\Gamma(G)$ contains a triangle, a contradiction. Thus $3 \nmid|N|$ and $|P|=3$, as claimed.

Step 2. Notice that $G=N P, N \cap P=1$. We claim that $G$ is a Frobenius group with Frobenius kernel $N$ and Frobenius complement $P$. Let $\psi \in \operatorname{Irr}(G)-\operatorname{Irr}(G / N)$. Then $k e r \psi \leq N$ since $N$ is the unique maximal normal subgroup of $G$. By Step 1, we have $3 \nmid|N|$ and hence $3 \nmid \mid$ ker $\psi \mid$. Since $3\left|a\left(\chi_{2}\right), 3\right| a\left(\chi_{3}\right)$ and $\Gamma(G)$ contains no triangles, we conclude that $3 \nmid a(\psi)=\frac{|G|}{|\operatorname{ker} \psi| \psi(1)}$. Hence $3 \nmid \frac{|G|}{\psi(1)}$ since $3 \nmid \mid$ ker $\psi \mid$. Therefore, by Lemma 2.5, for any 
$1 \neq x \in P$, we obtain $\psi(x)=0$. By the second orthogonality relation, we have $\left|C_{G}(x)\right|=$ $\sum_{\chi \in \operatorname{Irr}(G)} \chi(x) \chi\left(x^{-1}\right)=\sum_{\chi \in \operatorname{Irr}(G / N)} \chi(x) \chi\left(x^{-1}\right)=3=|P|$. But since $|P|=3, C_{G}(x) \geq P$ and thus $C_{G}(x)=P$ for any $1 \neq x \in P$. Therefore, $G$ is a Frobenius group with Frobenius kernel $N$ and Frobenius complement $P$, as claimed.

Step 3. We claim that $N$ is a $q$-group for some prime $q$. Otherwise, we may assume that $N=Q \times R$ where $Q$ and $R$ are the Sylow $q$ - and $r$ - subgroups of $N$ respectively by lemma 2.6 (the proof is similar if $|N|$ has more than two prime divisors). Since $|P|=3$ and $G$ is a Frobenius group by the Step 2, either $q \geq 5$ or $r \geq 5$. We may assume $r \geq 5$. By Step 2, $G$ is a Frobenius group with Frobenius complement $P$ of order 3 . This implies that every nonprincipal irreducible character of $N$ has precisely 3 distinct conjugates in $G$. Then we may choose irreducible characters $1_{R} \neq \xi_{i}(i=1,2)$ of $R$ such that $\xi_{1}$ and $\xi_{2}$ are not conjugate in $G$ since $R$ has at least 4 distinct nonprincipal irreducible characters.

Let $\theta \in \operatorname{Irr}(Q)$ such that $q \mid a(\theta)$. Then we have three irreducible characters of $N: \theta \times 1_{R}$, $\theta \times \xi_{1}$, and $\theta \times \xi_{2}$. It follows that $\left(\theta \times 1_{R}\right)^{G},\left(\theta \times \xi_{1}\right)^{G}$, and $\left(\theta \times \xi_{2}\right)^{G}$ are three distinct irreducible characters of $G$ since $G$ is a Frobenius group. By Lemmas 2.1] and 2.3, $q$ divides the codegrees of these three characters, which means that $\Gamma(G)$ has a triangle, a contradiction. Therefore, $N$ is a $q$-group for some prime $q$, as claimed.

Step 4. We claim that $q \leq 7$. Otherwise, suppose $q>7$. Since $|Z(N)| \geq q>7, N$ has at least 8 conjugacy classes. Hence $N$ has at least 7 nonprincipal irreducible characters whose codegrees are divided by $q$. Since $|P|=3$, there are at least 3 nonprincipal irreducible characters $\theta_{1}, \theta_{2}, \theta_{3}$ of $N$ which are not conjugate to each other in $G$ and $q|| a\left(\theta_{i}\right) \mid(1 \leq i \leq 3)$. Therefore, $\theta_{1}{ }^{G}, \theta_{2}{ }^{G}, \theta_{3}{ }^{G}$ are 3 distinct irreducible characters of $G$, and $q|| a\left(\theta_{i}{ }^{G}\right) \mid(1 \leq i \leq 3)$ by Lemma 2.1. which implies that $\Gamma(G)$ contains a triangle, a contradiction. Thus $q \leq 7$, as claimed.

Step 5. We claim that if $q=5$ or 7 , then $|N|=q$. Otherwise, suppose that $|N| \geq q^{2}$. If $N$ is abelian, then $N$ has at least $q^{2}>7$ conjugacy classes. If $N$ is nonabelian, then $|Z(N)| \geq q$ and $N$ has at least $q-1$ noncentral conjugacy classes. Thus $N$ has at least $2 q-1>7$ conjugacy classes. Therefore, whether $N$ is abelian or not, $N$ has at least 8 conjugacy classes and thus has at least 7 nonprincipal irreducible characters. By the same discussion as in Step 4, we obtain that $\Gamma(G)$ contains a triangle, a contradiction. Thus if $q=5$ or 7 , then $|N|=q$, as claimed.

Step 6. We claim $q \neq 5$. Since $G$ is a Frobenius group with Frobenius kernel $N$ and Frobenius complement $P$ of order $3,3 \mid(|N|-1)$. Thus, $q \neq 5$, as claimed.

Step 7. We claim that $|N|=4$ if $N$ is a 2-group. We may assume that $|N|=4^{n}$ for some positive integer $n$ since $3 \mid(|N|-1)$. 
Suppose $n=2$ and $|\operatorname{Irr}(N)| \geq 8$. Using the same discussion as in Step 4, we deduce that $\Gamma(G)$ contains a triangle, a contradiction. We now suppose $n=2$ and $|\operatorname{Irr}(N)| \leq 7$. Then $N$ is not abelian. By the character table of all nonisomorphic nonabelian groups of order 16 in section 25 of [4, there are exactly 3 distinct nonisomorphic nonabelian groups of order 16 who have at most 7 irreducible characters and they all have precisely three irreducible characters whose degrees are 2. Moreover, two of them are faithful and the other one is not faithful. So if $N$ is one of the 3 distinct nonisomorphic nonabelian groups of order 16, then the exact three irreducible characters of degree 2 can not be conjugate in $G$, which contradicts to the fact that $G$ is a Frobenius group with Frobenius complement $P$ of order 3 . Thus $n \neq 2$. So we may assume $n \geq 3$.

Indeed, we may further assume $|\operatorname{Irr}(N)| \leq 7$. Since $N$ is nilpotent, $N^{\prime}<N$. Note that $G$ is a Frobenius group with Frobenius complement $P$ of order 3 . Hence the degrees of irreducible characters of $N$ must be $1,1,1,1, y, y, y$ for some positive integer $y$. Therefore, we have $4+$ $3 y^{2}=4^{n}$. It is easy to see $y=2 z$ for some positive integer $z$. So $1+3 z^{2}=4^{n-1}$ and hence $\left(2^{n-1}+1\right)\left(2^{n-1}-1\right)=3 z^{2}$. Since $\left(2^{n-1}+1,2^{n-1}-1\right)=1$, we know $2^{n-1}+1=u^{2}$ or $2^{n-1}-1=u^{2}$ for some positive integer $u$. But since $n \geq 3,2^{n-1}-1 \equiv 3(\bmod 4)$, and $u^{2} \equiv 0$ or $1(\bmod 4)$, it follows that $2^{n-1}-1 \neq u^{2}$. Hence we have $2^{n-1}+1=u^{2}$, which means that $(u+1)(u-1)=2^{n-1}$. Thus $u+1$ and $u-1$ are powers of 2 . Note that $(u+1)-(u-1)=2$. Hence it follows that $u-1=2, u=3$, and $n=4$. But this leads to $z^{2}=21$, a contradiction. Thus the claim holds.

Step 8. Now we show that $N$ is a 2 -group of order 4 or a cyclic group of order 7 . If $N$ is the latter case, $G$ is the nonabelian group $F_{7,3}$ of order 21 . Notice that the nonprincipal irreducible character codegrees of $F_{7,3}$ are $3,3,7,7$. The graph $\Gamma\left(F_{7,3}\right)$ does contain no triangles.

So we may assume that $N$ is a 2 -group of order 4 . Then $|G|=12$. By the character table of all 3 nonisomorphic nonabelian groups of order 12 in [4, the nonprincipal irreducible character codegrees of $A_{4}$ are $3,3,4$ and $A_{4}$ is the only nonabelian group of order 12 whose character graph associated with codegrees contains no triangles. Thus the proof of the lemma is finished.

Lemma 4.6 Let $G$ be a nonabelian finite group such that $\Gamma(G)$ has no triangles. If $G$ has a normal subgroup $N$ such that $|G: N|=2$, then $G$ is isomorphic to the symmetric group $S_{3}$ or the dihedral group $D_{10}$ of order 10 .

Proof. Note that $N \neq 1$ since $G$ is nonabelian. Let $\operatorname{Irr}(G / N)=\left\{\chi_{1}=1_{G}, \chi_{2}\right\}$ and $P \in S y l_{2}(G)$. Then $2 \mid a\left(\chi_{2}\right)$. By Lemma 4.4, we may assume that $G^{\prime}=N$ and that $N$ is the unique maximal normal subgroup of $G$.

Step 1. We claim that for any $\chi \in \operatorname{Irr}(G)-\operatorname{Irr}(G / N), \chi(1)$ is even. Otherwise, suppose that there is a $\psi_{1} \in \operatorname{Irr}(G)-\operatorname{Irr}(G / N)$ such that $\psi_{1}(1)$ is an odd integer. Since 2||$G \mid$ and 
$\sum_{\chi \in \operatorname{Irr}(G)-\operatorname{Irr}(G / N)} \chi(1)^{2}=|G|-2$, there is another $\psi_{2} \in \operatorname{Irr}(G)-\operatorname{Irr}(G / N)$ such that $\psi_{2}(1)$ is an odd integer. Note that $\operatorname{ker} \psi_{i} \leq N(1 \leq i \leq 2)$ since $N$ is the unique maximal normal subgroup of $G$ and that $|G: N|=2$. Therefore, we have $2 \mid \frac{|G|}{\left|k e r \psi_{i}\right|}$. But since $2 \nmid \psi_{i}(1)$, it follows that $2 \mid \frac{|G|}{\mid \text { ker } \psi_{i} \mid \psi_{i}(1)}$ and $2 \mid a\left(\psi_{i}\right)(1 \leq i \leq 2)$. This implies that $\Gamma(G)$ contains a triangle consisting of $\psi_{1}, \psi_{2}$ and $\chi_{2}$, a contradiction. Thus the claim holds.

Step 2. We claim that $G$ is a Frobenius group with Frobenius kernel $N$ and Frobenius complement $P$. By the claim of Step 1,

$$
|G|=\sum_{\chi \in \operatorname{Irr}(G)} \chi(1)^{2}=\sum_{\chi \in \operatorname{Irr}(G)-\operatorname{Irr}(G / N)} \chi(1)^{2}+2 \equiv 2(\bmod 4) .
$$

This means that $|G| / 2$ is an odd integer. Hence $|P|=2$ and $2 \nmid \frac{|G|}{\psi(1)}$ for any $\psi \in \operatorname{Irr}(G)-$ $\operatorname{Irr}(G / N)$. By Lemma 2.5, for any $1 \neq x \in P, \psi \in \operatorname{Irr}(G)-\operatorname{Irr}(G / N)$, we have $\psi(x)=0$. Therefore, by the second orthogonality relation, $\left|C_{G}(x)\right|=\sum_{\chi \in \operatorname{Irr}(G)} \chi(x) \chi\left(x^{-1}\right)=\sum_{\chi \in \operatorname{Irr}(G / N)} \chi(x) \chi\left(x^{-1}\right)=$ $2=|P|$. This means that $C_{G}(x)=P$ for any $1 \neq x \in P$. Thus $G$ is a Frobenius group with Frobenius kernel $N$ and Frobenius complement $P$, as claimed.

Step 3. By Lemma 2.6, $N$ is abelian. Using the similar argument as in Steps 3, 4, and 5 in Lemma 4.5. we deduce that $|N| \leq 5$ and thus $N$ is a cyclic group of order 3 or 5 . Therefore $G$ is isomorphic to the symmetric group $S_{3}$ or the dihedral group $D_{10}$ of order 10 .

Now we can prove Theorem 1.4

Proof of Theorem 1.4 Let $G$ be a group appeared in the theorem. By Lemmas 4.3, 4.4, 4.5. and 4.6, it suffices to show that $\Gamma(G)$ contains no triangles. This is obvious if $G$ is a cyclic group of order 2 or 3 . For other cases, it is shown by the following table:

\begin{tabular}{|l|l|}
\hline Group & Nonprincipal irreducible character codegrees \\
\hline$S_{3}$ & 2,3 \\
\hline$D_{10}$ & $2,5,5$ \\
\hline$A_{4}$ & $3,3,4$ \\
\hline$F_{7,3}$ & $3,3,7,7$ \\
\hline
\end{tabular}

\section{Acknowledgement}

We really appreciate the encouragement of Prof. Jiping Zhang. 


\section{References}

[1] Y. Berkovich and E. Zhmud', Characters of finite groups, Part 1, 2, American Mathematical Society, Providence, R.I., 1997.

[2] D. Chillag, A. Mann, and O. Manz, The co-degrees of irreducible characters, Israel J. Math. 73(1991) 207-223.

[3] I. M. Isaacs, Character Theory of Finite Groups, Academic Press, New York, 1976.

[4] G. James, M. Liebeck, Representations and Characters of Groups, Cambridge University Press, Cambridge, 2001.

[5] M. L. Lewis, An overview of graphs associated with character degrees and conjugacy class sizes in finite groups, Rocky Mountain J. Math. 38 (1)(2008) 175-211.

[6] T. Li, Y. Liu, and X. Song, Finite nonsolvable groups whose character graphs have no triangles, J. Algebra 323(2010) 2290-2300.

[7] G. Qian, A note on element orders and character codegrees, Arch. Math. 97(2011) 99-103.

[8] G. Qian, Y. Wang, and H. Wei, Co-degrees of irreducible characters in finite groups, J. Algebra 312(2007) 946-955.

[9] J. Rose, A Course on Group Theory, Cambridge University Press, Cambridge, 1978.

[10] J. G. Thompson, Normal p-complements for finite groups, Math. Z. 72(1960) 332-354.

[11] Y. Wu, P. Zhang, Finite solvable groups whose character graphs are trees, J. Algebra 308 (2)(2007) 536-544.

[12] J. Zhang, A note on character degrees of finite solvable groups, Comm. Algebra 28 (9)(2000) 4249-4258.

[13] J. Zhang, Finite solvable groups whose character degree graphs are not complete, Algebra Colloq. 13 (4)(2006) 541-552. 